\title{
Amino Terminus of Substance P Potentiates Kainic Acid-induced Activity in the Mouse Spinal Cord
}

\author{
Alice A. Larson and Xiaofeng Sun \\ Department of Veterinary Pathobiology, University of Minnesota, Saint Paul, Minnesota 55108
}

Sensitization to the behavioral effects produced by repeated injections of kainic acid (KA) into the mouse spinal cord area has been previously shown to be abolished by pretreatment with capsaicin, a neurotoxin of substance P (SP)-containing primary afferent $\mathrm{C}$-fibers. While SP has a variety of well characterized biological actions that are mediated by interactions of its $\mathrm{COOH}$ terminus with neurokinin receptors, more recently we have characterized an amino-terminally directed SP binding site. The present studies were initiated to determine whether behavioral sensitization to repeated injections of intrathecally administered KA is mediated by the COOH or $\mathrm{NH}_{2}$ terminal of SP. In the present studies, pretreatment with SP(1-7), an $\mathrm{NH}_{2}$-terminal fragment of SP, but not SP(5-11), a COOH-terminal fragment, potentiated KA-induced behavioral activity in mice. Pretreatment with [D-Pro ${ }^{2}$, D-Phe ${ }^{r}$ SP(1-7), an inhibitor of SP $\mathbf{N H}_{2}$-terminal binding, blocked the potentiative effect of $\operatorname{SP}(1-7)$ as well as the sensitization to repeated injections of KA. In contrast, [DPro $\left.^{2}, \mathrm{D}-\mathrm{Trp}^{7,9}\right] \mathrm{SP}$, a neurokinin antagonist, had little effect on behavioral sensitization to KA. The present study suggests that SP has an important modulatory role on excitatory amino acid activity in the spinal cord that is mediated by an action of the $\mathrm{NH}_{2}$ terminal of SP at a non-neurokinin receptor.

Substance $\mathrm{P}$ (SP) and endogenously occurring excitatory amino acids (EAAs), such as glutamate and aspartate, have been proposed to mediate pain transmission in the spinal cord (Lembeck, 1953; Salt and Hill, 1983; Besson and Chaouch, 1987). Immunohistochemical studies have shown that both glutamateand SP-like immunoreactivity are colocalized in small-diameter primary afferent neurons (De Biasi and Rustioni, 1988). Further, nociceptive stimulation evokes the release of both EAAs in the spinal cords of conscious, freely moving rats (Skilling et al., 1988) and of SP in the spinal cords of decerebrate rats (Takagi, 1984). If both SP and EAAs are important mediators of pain transmission at the level of the spinal cord, the possibility exists that nociceptive activity is mediated by an interaction between SP and EAA neural circuits.

Substantial evidence exists for an interaction between SP and FAAs in the transmission of noxious stimuli (Dougherty and

Received Apr. 16, 1992; revised July 8, 1992; accepted July 10, 1992,

We thank Drs. Virginia S. Seybold, Alvin J. Beitz, Darrell Mousseau, and David $R$. Brown for their editorial assistance in the preparation of the manuscript. This work was supported by U.S. Public Health Service Grants NIDA00124, DA04090, and DA04190.

Correspondence and reprint requests should be addressed to Dr. Alice A. Larson, Department of Veterinary Pathobiology, University of Minnesota, 295 Animal Science/Veterinary Medicine Building, 1988 Fitch Avenue, St. Paul, MN 55108. Copyright (C) 1992 Society for Neuroscience $0270-6474 / 92 / 124905-06 \$ 05.00 / 0$
Willis, 1991) as well as spinally mediated motor activity (Sun and Larson, 1991). EAAs and SP each produce caudally directed biting and scratching (CBS) behavior when injected intrathecally in mice (Hylden and Wilcox, 1981; Piercy et al., 1981; Aanonsen and Wilcox, 1987). Using this approach, injections of SP at 2 min intervals result in a decreased responsivity to the behavioral effects of SP (Larson, 1988). In an identical injection protocol, kainic acid (KA), a non-NMDA EAA, produced behavioral responses of progressively greater intensity after repeated injections (Sun and Larson, 1991). While repeated injections of SP resulted in an attenuated behavioral response, pretreatment with SP significantly enhanced the behavioral response to a subsequent injection of KA. In contrast, depletion of SP in primary afferent fibers of the spinal cord using capsaicin prevented the development of sensitization to repeated injections of KA (Sun and Larson, 1991). Pretreatment with capsaicin does not alter the magnitude of response to a single injection of KA, suggesting that SP mediates the increase in CBS behaviors after repeated injections of KA, but not the response to a single injection of KA (Sun and Larson, 1991).

The ability of SP to induce CBS behaviors through interaction of its $\mathrm{COOH}$ terminus with neurokinin receptors is widely recognized and is readily antagonized by neurokinin antagonists (Sakurada et al., 1989). $\mathrm{NH}_{2}$-terminal fragments of SP are inactive at neurokinin receptors but have been found to be active in the CNS (Stewart et al., 1982; Hall and Stewart, 1983, 1984) and bind to high-affinity binding sites in the mouse brain and spinal cord (Igwe et al., 1990c). The effect of SP $\mathrm{NH}_{2}$-terminal fragments on EAA-induced activity has not been previously explored. The present studies were initiated to determine whether SP facilitates KA-induced behavior by an action at either the neurokinin or SP $\mathrm{NH}_{2}$-terminal receptor and to test the hypothesis that behavioral sensitization to $\mathrm{KA}$ is mediated by such an action of SP. We examined the ability of $\mathrm{COOH}$ - and $\mathrm{NH}_{2}$ terminal fragments of SP to potentiate the behavioral action of $\mathrm{KA}$, as well as the ability of selective blockers either of neurokinin receptors or of $\mathrm{SP} \mathrm{NH}_{2}$-terminal binding sites to prevent potentiation of KA-induced CBS behavior in mice.

\section{Materials and Methods}

Animals. Male Swiss-Webster mice (20-25 gm; Bio-Lab, White Bear Lake, MN, and Sasco Inc., Omaha, NE) were housed four per cage and allowed to acclimate for at least $24 \mathrm{hr}$ prior to use. Mice were allowed free access to food and water and used strictly in accordance with the Guidelines of the University of Minnesota Animal Care and Use Committee and those prepared by the Committee on Care and Use of Laboratory Animals of the Institute of Laboratory Animal Resources, National Research Council [DHEW publication (NIH) 78-23, revised 1978].

Drug administration. Mice were injected intrathecally as described previously (Hylden and Wilcox, 1980) into the subarachnoid space at 

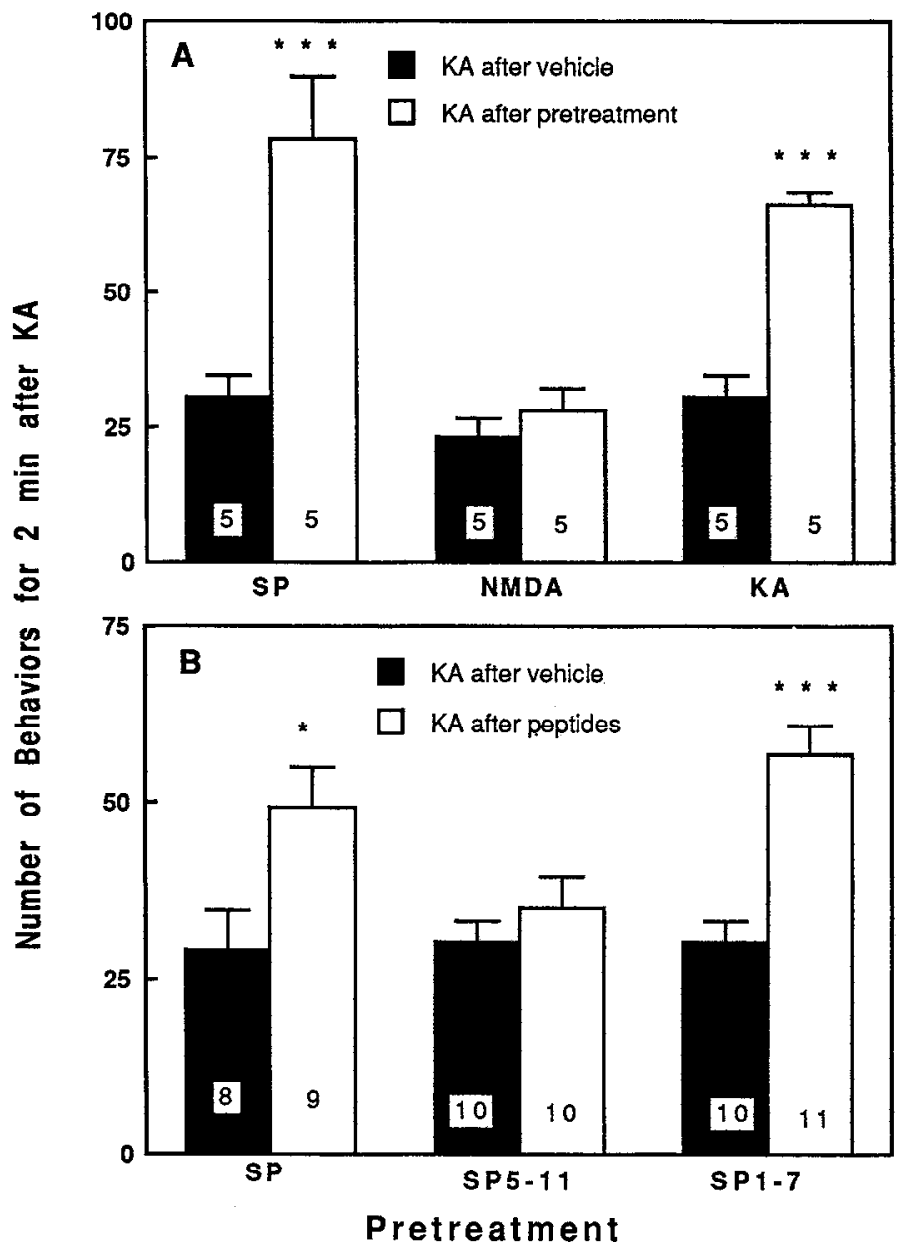

Figure 1. Intensity of KA-induced behavioral responses in mice after pretreatment with SP, KA, or NMDA $(A)$ and after pretreatment with SP peptide fragments $(R)$. Data summarizing CBS responses throughout represent the mean number $( \pm S E M)$ of behaviors produced during a 2 min interval immediately following an intrathecal injection of KA. Pretreatment denotes the compound injected intrathecally three times at 2 min intervals prior to KA. Effects were compared to those in vehicleinjected mice tested on the same day. Drugs were administered in the following doses per injection: 7.5 pmol of SP, 200 pmol of NMDA, 25 pmol of KA, $7.5 \mathrm{pmol}$ of SP peptide fragments. Numbers at the base of each bar are the number of animals used in each group. Throughout the figures, asterisks indicate a significant difference between the test and vehicle-injected control groups $\left({ }^{*}, p<0.05 ;^{* * *}, p<0.001\right)$ using the Student's $t$ test.

approximately the L5-L6 intervertebral space of the spinal cord. Injections were accomplished using a needle inserted into PE-10 tubing about $20 \mathrm{~cm}$ long, fitted with a second 30-gauge needle tip that is ultimately inserted into the subarachnoid space, which allows repeated delivery of drug into the same spinal area (Larson and Beitz, 1988). The cannula is completely filled with drug solutions prior to injection to avoid injection of air.

Drugs. Kainic acid and NMDA were purchased from Sigma (St. Louis, MO). Substance P (SP), SP(5-11), SP(1-7), phosphoramidon, and [D-Pro ${ }^{2}$, D-Trp ${ }^{7,9}$ SP (DPDT-SP) were purchased from Peninsula Laboratories (Belmont, CA). [D-Pro ${ }^{2}$, D-Phe $]$ SP(1-7) [D-SP(1-7)] was synthesized by the University of Minnesota Microchemical Facilities (Minneapolis, MN). All drugs were dissolved in acidified saline composed of $0.85 \% \mathrm{NaCl}$ containing $0.01 \mathrm{~N}$ acetic acid, pH 2.0 (Hall and Stewart, 1986), except KA and NMDA, which were dissolved in normal saline. Acidified saline was also used for vehicle-injected controls for each drug where appropriate.

Experimental protocol. After insertion of the intrathecal cannula, mice were observed for $1 \mathrm{~min}$ prior to drug injection to assess normal exploratory behavior and locomotion. KA was injected at a dose of 25
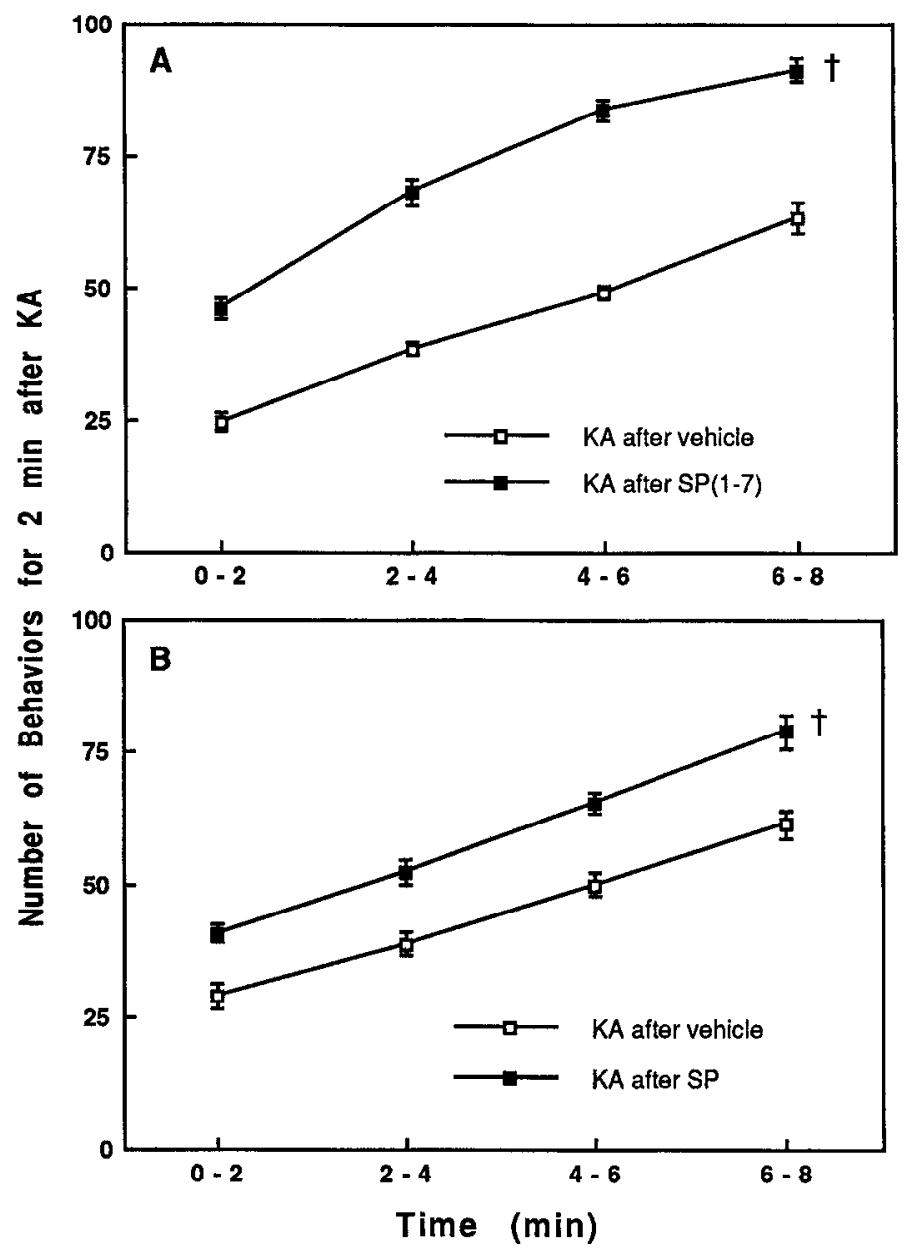

Figure 2. Behavioral sensitization to the CBS effects produced by 25 pmol of KA injected intrathecally at 2 min intervals and the ability of three intrathecal injections of either $7.5 \mathrm{pmol}$ of SP(1-7) $(A)$ or $7.5 \mathrm{pmol}$ of $\mathrm{SP}(B)$, at $2 \mathrm{~min}$ intervals, to potentiate $\mathrm{KA}$ responses compared to vehicle-injected control mice. Each point represents the mean number $( \pm$ SEM) of behaviors from six mice in cach group. $\dagger$, a statistically significant effect of peptide pretreatment on KA responses using ANO$\mathrm{VA}$ and $p<0.05$ as the level of significance.

pmol in a $5 \mu \mathrm{l}$ vol, which produced CBS behaviors that are similar to those observed after intrathecally injected SP. The intensity of the behavioral response, in terms of number of caudally directed head pokes, bites, or scratches or continuous caudally directed grooming, at a rate of one count per second, was quantified over a 2 min interval immediately following injection of KA. Injections of acidified saline or SP(17) elicited no CBS behaviors and appeared to have no effect on the normal exploratory behavior in mice. All observations were made during the $12 \mathrm{hr}$ light cycle of the mouse facility.

Statistics. Group averages and SEM are reported. Statistical analysis of the results was performed using Student's $t$ test for unpaired or paired samples or using ANOVA, as indicated. In each analysis, the control and test groups were considered to be significantly different when $P$ values were less than 0.05 .

\section{Results}

Selectivity of SP-mediated potentiation of $K A$. The behavioral response to a single injection of KA, SP, NMDA, or SP(5-11), at the doses administered, is immediate in onset and lasts for less than 2 min. As reported previously (Sun and Larson, 1991), repeated injections of KA resulted in a progressive increase in the intensity of behaviors elicited in response to each subsequent administration of KA at 2 min intervals (Fig. $1 A$ ). Enhancement of KA activity was also produced by pretreatment with three 


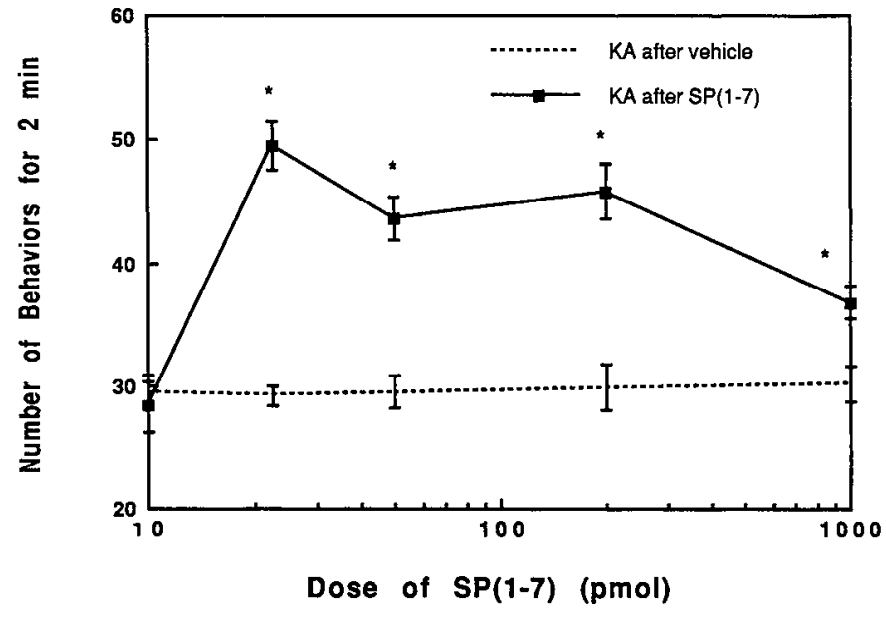

Figure 3. The effect of a single bolus injection of SP(1-7) on CBS behaviors induced by KA. Values indicate the mean number ( \pm SEM) of CBS behaviors produced over a 2 min interval following the intrathecal injection of $25 \mathrm{pmol}$ of KA $30 \mathrm{~min}$ after either vehicle or various doses of $\mathrm{SP}(1-7)$. Values obtained from control mice injected with vehicle rather than $\mathrm{SP}(1-7)$ are also shown for comparison as indicated by the broken line. A minimum of six mice were used in each group.

injections of $7.5 \mathrm{pmol}$ of SP at 2 min intervals immediately prior to KA (Fig. $1 A$ ). The ability of SP to enhance KA-induced behaviors was selective among excitatory compounds, as pretreatment with three injections of 200 pmol of NMDA failed to alter the behavioral response to KA (Fig. $1 A$ ).

To ascertain the portion of the SP peptide necessary to enhance KA-induced activity, the potentiative effect of SP itself was compared to the effect of $\mathrm{COOH}$ - and $\mathrm{NH}_{2}$-terminal heptapeptide fragments of SP (Fig. $1 B$ ). Pretreatment with three injections of 7.5 pmol of SP(5-11), a COOH-terminal fragment that retains its behavioral activity at neurokinin receptors (Hall and Stewart, 1983, 1984), resulted in short episodes of CBS behavior immediately following each injection of SP(5-11), which subsided prior to injection of KA. While SP(1-7) produced no behavioral response when injected alone, pretreatment with SP(1 7), but not $\mathrm{SP}(5-11)$, potentiated the behavioral response to subsequent injection of $\mathrm{KA}$.

Additivity of $S P$ - and $K A$-induced potentiation of $K A$. Pretreatment with three injections of 7.5 pmol of either SP(1-7) or SP 2 min prior to four injections of $\mathrm{KA}$ each enhanced the intensity of behavioral responses to each injection of KA (Fig. 2). The enhancement of KA-induced activity due to pretreatment with either SP or SP(1-7) resulted in a parallel shift in the intensity of behavioral responses over time after SP and after SP(1-7) compared to those after vehicle.

Time course and dose-response characteristics of potentiation of $K A$ by $S P(1-7)$. When injected at various intervals prior to KA administration, the effect of a single bolus injection of 22.5 pmol of SP(1-7) was to enhance the behavioral response to KA injected 15-90 min after SP(1-7). Response to KA $2 \mathrm{hr}$ after this dose of $\operatorname{SP}(1-7)$ did not differ from that after vehicle (data not shown). When injected $30 \mathrm{~min}$ prior to $\mathrm{KA}$, a time at which potentiation is observed, doses of $\mathrm{SP}(1-7)$ ranging from 10 to $1000 \mathrm{pmol}$ of SP(1-7) resulted in an inverted U-shaped doseresponse curve for potentiation of KA (Fig. 3), suggesting the recruitment of an additional, unidentified action produced by high doses of SP(1-7).

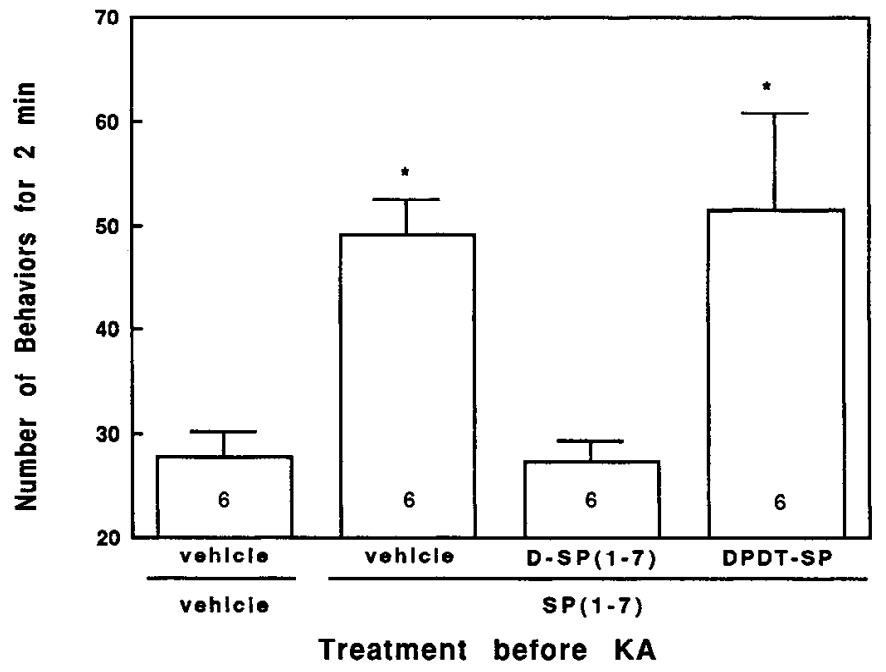

Figure 4. D-SP(1-7) inhibits whereas DPDT-SP does not affect the ability of $S P(1-7)$ to potentiate KA-induced behavioral activity. The effect of DPDT-SP or D-SP(1-7), an SP(1-7) antagonist, on the ability of $S P(1-7)$ to potentiate the behavioral response to a single intrathecal injection of $25 \mathrm{pmol}$ of $\mathrm{KA}$ was examined $30 \mathrm{~min}$ after the intrathecal injection of $22.5 \mathrm{pmol}$ of SP(1-7). A dose of $1.1 \mathrm{nmol}$ of D-SP(1-7), $0.74 \mathrm{nmol}$ of DPDT-SP, or vehicle was injected intrathecally $5 \mathrm{~min}$ prior to KA. Neither antagonist significantly altered the basal activity produced by the injection of KA alone.

Effect of $\mathrm{COOH}$ - and $\mathrm{NH}_{2}$-terminal antagonists of SP. DPDTSP, a neurokinin antagonist (Engberg et al., 1981), and D-SP(17), a compound that inhibited $\left[{ }^{3} \mathrm{H}\right] \mathrm{SP}(1-7)$ binding in mouse brain membranes (Igwe et al., 1990c), were used to assess the influence of the two SP receptors on the development of behavioral sensitization to KA. DPDT-SP and D-SP(1-7) were first tested by assessing their degree of selectivity on SP-induced CBS behavior caused by activation of neurokinin receptors, compared to their effect on the ability of SP(1-7) to potentiate KAinduced CBS behavior. The average number of CBS behaviors induced by 11.25 pmol of SP 5 min after vehicle $(63.3 \pm 1.2$; $n=6)$ was inhibited by pretreatment $(5 \mathrm{~min})$ with $0.74 \mathrm{nmol}$ of DPDT-SP $(25.7 \pm 3.9 ; n=6)$. In contrast, $1.1 \mathrm{nmol}$ of $\mathrm{D}-\mathrm{SP}(1-7)$ had no effect on the intensity of SP-induced CBS behaviors $(63.5 \pm 1.8 ; n=6)$, indicating that $\mathrm{D}-\mathrm{SP}(1-7)$ is devoid of neurokinin antagonistic activity. While $\mathrm{D}-\mathrm{SP}(1-7)$ had no effect on SP-induced behaviors, pretreatment of mice with D-SP(1-7) (1.1 nmol, $5 \mathrm{~min})$ completely blocked the potentiative effect of $\mathrm{SP}(1-7)(22.5 \mathrm{pmol}, 30 \mathrm{~min}$ prior to testing) on the behaviors elicited by KA (Fig. 4) without altering the response to KA itself (not shown). Pretreatment with $0.74 \mathrm{nmol}$ of DPDTSP 5 min prior to testing with KA (not shown) did not significantly alter either the behavioral effect of KA or the potentiative effect of SP(1-7) on KA-induced activity (Fig. 4).

Pretreatment of mice with $0.74 \mathrm{nmol}$ of DPDT-SP, $5 \mathrm{~min}$ prior to challenge with repeated injections of $\mathrm{KA}$, inhibited the development of sensitization to the behavioral effects of $\mathrm{KA}$ to a small but significant degree (Fig. 5). In contrast, the effect of $1.1 \mathrm{nmol}$ of $\mathrm{D}-\mathrm{SP}(1-7) 5 \mathrm{~min}$ prior to KA was a complete blockade of the development of sensitization to repeated injections of KA (Fig. 5). The injection of these SP antagonists, either alone or in combination with other drugs, did not impair motor activity at the doses used.

Inhibition of endopeptidase activity. Pretreatment of mice with 3 nmol of phosphoramidon, an endopeptidase inhibitor, in- 
Figure 5. Inhibitory effects of 0.74 nmol of DPDT-SP, a neurokinin antagonist, and $1.1 \mathrm{nmol}$ of $\mathrm{D}-\mathrm{SP}(1-7)$ on sensitization to the behavioral effects of $25 \mathrm{pmol}$ of KA. Antagonists or saline were administered intrathecally $5 \mathrm{~min}$ prior to KA. Each point represents the mean number ( \pm SEM) of CBS behaviors produced for a 2 min interval immediately following the intrathecal injection of KA. Twelve mice were used in groups pretreated with antagonists, and 24 mice are represented by the control curve. The effects produced by antagonists were statistically compared to an equal number of control mice tested on the same day using ANOVA and $p$ $<0.05$ as the level of significance. Control data were then pooled for graphical representation.

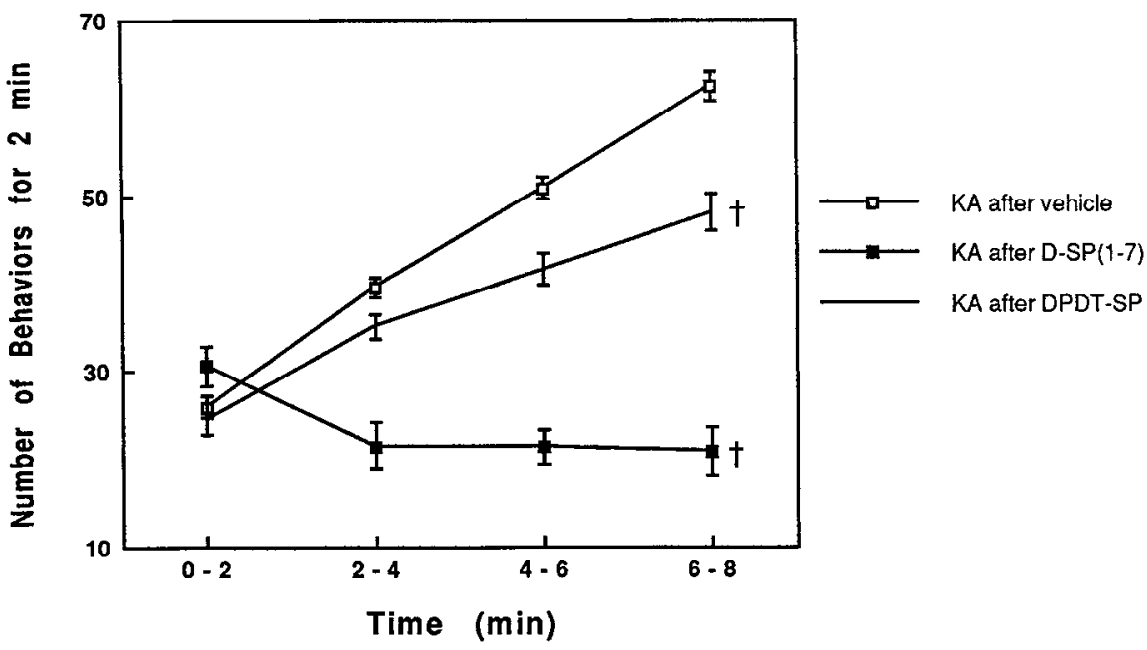

jected slowly over an 8 min interval immediately prior to injection of KA, resulted in an enhancement of KA-induced activity (Fig. 6). Pretreatment with either $0.74 \mathrm{nmol}$ of DPDTSP or $1.1 \mathrm{nmol}$ of D-SP(1-7) immediately prior to phosphoramidon and 8 min prior to injection of the first challenge with $\mathrm{KA}$ potentiated the response to $\mathrm{KA}$ after phosphoramidon in a fashion identical to that in the absence of peptidase inhibitors, described above. Just as in the absence of phosphoramidon, after phosphoramidon there was a small inhibition of behavioral sensitization after DPDT-SP and a dramatic inhibition of the development of sensitization to repeated injection of KA after D-SP(1-7) (Fig. 6.).

\section{Discussion}

The present data support the hypothesis that endogenously released SP facilitates KA-induced behavioral activity by an action mediated primarily by the $\mathrm{SP} \mathrm{NH}_{2}$ terminus and only to a minor extent by an action of the $\mathrm{COOH}$ terminus at neurokinin receptors. The ability of SP to potentiate KA-induced activity can be mimicked by exogenously administered SP or SP(1-7) but not $\operatorname{SP}(5-11)$.

The ability of capsaicin pretreatment to inhibit the development of behavioral sensitization to KA (Sun and Larson, 1991 ) suggests mediation of this phenomenon by SP. This possibility was recently supported by the observation that KA is capable of enhancing the release of SP from the dorsal spinal cords of conscious, freely moving rats, as measured using in vivo microdialysis (Murray et al., 1990). The ability of DPDTSP to inhibit the development of sensitization to the behavioral effects of KA to a small but significant degree (Fig. 5) suggests that a small component of the enhanced response to KA may be mediated by activity at neurokinin receptors. In contrast, injection of D-SP(1-7), which selectively inhibited the ability of the exogenously administered $\mathrm{SP} \mathrm{NH}_{2}$-terminal fragment to potentiate KA but not the neurokinin behavioral activity elicited by $\mathrm{SP}$, completely blocked the development of sensitization to repeated injections of KA. These data support the concept that sensitization to the behavioral effects of repeated injections of $\mathrm{KA}$ appears to result primarily from activation of receptors selective for the $\mathrm{NH}_{2}$-terminal portion of the SP peptide.

If the mechanism underlying behavioral sensitization to $\mathrm{KA}$ is the same as that mediating the enhancement of KA activity by either SP or SP(1-7), the two effects would be expected to produce a potentiation of KA-induced behavior that is no greater than the sum of each potentiative effect alone. If behavioral sensitization to KA results from a distinct mechanism from that underlying the potentiation of KA by pretreatment with $\mathrm{SP}(1$ 7), their combined potentiative effects may enhance the response to KA to a greater degree than that produced by pretreatment with either KA or SP(1-7) alone. To test these possibilities, we
Figure 6. Potentiation of the behavioral response to four injections of 25 pmol of KA by pretreatment with 3 nmol of phosphoramidon injected slowly over an $8 \mathrm{~min}$ period immediately prior to the intrathecal injection of KA. D-SP(1-7) and DPDT-SP were administered as described in Figure 5. Each point represents the mean number $( \pm$ SEM) of CBS behaviors from groups of at least six mice, analyzed and pooled as indicated in Figure $5 . \dagger$, a significant inhibition of behaviors in phosphoramidon-injected mice by $\mathrm{D}-\mathrm{SP}(1-7)$ and DPDT-SP.

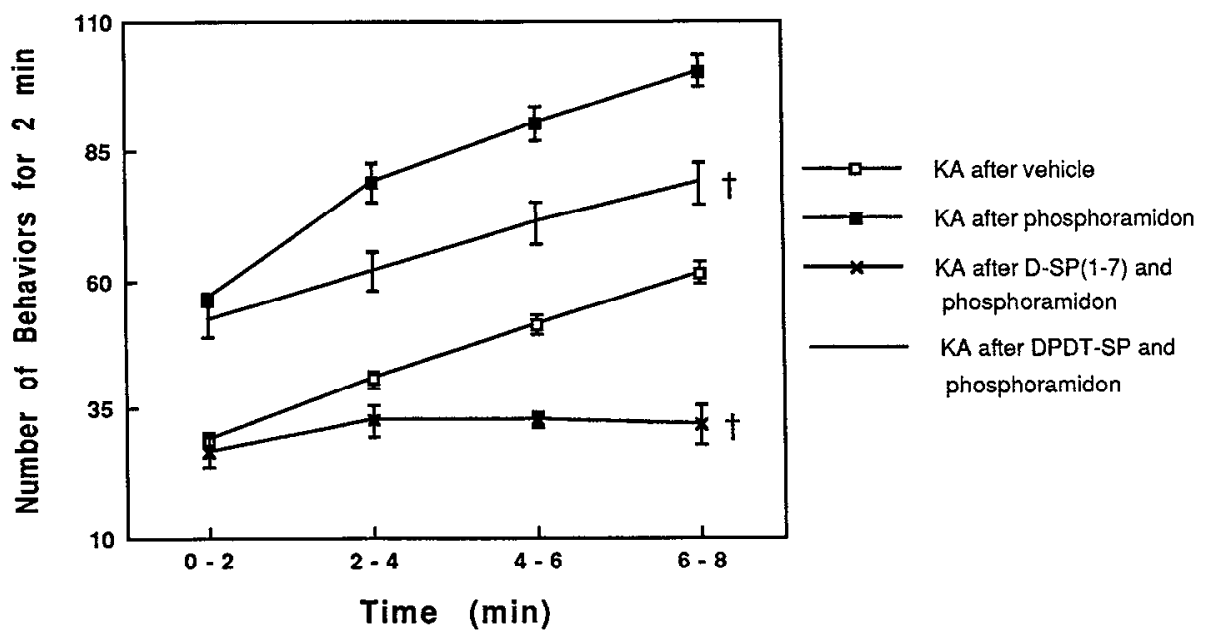


measured the effect of pretreatment with SP(1-7) on the development of behavioral sensitization to four injections of KA. Our results suggest that either SP or SP(1-7) is capable of enhancing $\mathrm{KA}$-induced activity, resulting in a parallel shift in the intensity of response to each injection of KA (Fig. 2). The degree of behavioral sensitization to KA, measured by the average increase in the intensity of behaviors from the first to the fourth injection of KA, was not affected by either peptide. These data are consistent with a simple additivity of the effects of pretreatment with KA or SP(1-7) and suggest a common mechanism mediating their potentiation of KA activity.

In a variety of species, SP is metabolized rapidly in the extracellular space by endopeptidases (Nyberg et al., 1984; Sakurada et al., 1985; Igwe et al., 1990b), which are sensitive to nonspecific peptidase inhibitors, such as phosphoramidon. If behavioral sensitization to $\mathrm{KA}$ is mediated primarily by the intact, nonmetabolized SP molecule, pretreatment with peptidase inhibitors would be expected to potentiate sensitization. If sensitization is mediated exclusively via the generation of $\mathrm{NH}_{2}$ terminal metabolites of SP, the addition of peptidase inhibitors should attenuate sensitization to KA. Our data show that pretreatment of mice with phosphoramidon resulted in an enhancement of behavioral activity after the first injection of KA (Fig. 6), consistent with a role for endopeptidase-sensitive peptides in the mediation of the behavioral effect of KA. The failure of phosphoramidon to block the development of sensitization to KA also suggests that metabolism of the intact SP molecule to $\mathrm{SP} \mathrm{NH}_{2}$-terminal peptide fragments is not necessary to potentiate KA-induced activity. That DPDT-SP and D-SP(1-7) affected the enhanced response to $\mathrm{KA}$ after phosphoramidon in a fashion identical to that in the absence of peptidase inhibitors (Fig. 6) suggests that potentiation by phosphoramidon is brought about by the same peptide as that mediating sensitization to $\mathrm{KA}$ in the absence of the peptidase inhibitor. Together, these data support the importance of the $\mathrm{NH}_{2}$-terminal amino acid sequence of the intact SP molecule in the facilitation of KA activity.

The prevention of behavioral sensitization to KA by pretreatment with capsaicin initially suggested that sensitization to KA is brought about by the release of an endogenous transmitter contained in primary afferent C-fibers (Sun and Larson, 1991). Based on the ability of SP, like KA, to evoke CBS behavior in the mouse spinal cord, it seemed reasonable to hypothesize that the behavioral sensitization was a simple additive effect of nonNMDA receptor activity and neurokinin activity. The present results suggest, however, that an action of the $\mathrm{NH}_{2}$ terminus of $\mathrm{SP}$, which induces no CBS behavioral response by itself, is a more likely mediator of behavioral sensitization to KA. The ability of $\mathrm{NH}_{2}$-terminal SP fragments, like SP(1-7), and the $\mathrm{NH}_{2}$ terminal amino acid sequence of the intact SP peptide to potentiate $\mathrm{KA}$-induced activity is a novel action that may prove to be important in a variety of systems throughout the CNS. One might speculate that the $\mathrm{NH}_{2}$ terminus of SP may be responsible for activity evoked by SP in areas of the CNS, such as the substantia nigra, that contain low concentrations of neurokinin receptors but high densities of SP-immunoreactive nerve terminals.

The ability of capsaicin to inhibit behavioral sensitization to KA (Sun and Larson, 1991) suggests an involvement of the same pool of SP as in nociceptive processing, which is also sensitive to capsaicin. The hyperalgesic activity of SP on mechanically induced nociception has also been found to be insensitive to reversal by DPDT-SP (Matsumura et al., 1985), suggesting that the role of SP in nociception may not be completely dependent on neurokinin receptor activation. One might speculate that facilitation of EAAs by the $\mathrm{NH}_{2}$-terminal portion of SP plays a role in nociceptive processing. Injection of $\mathrm{SP}(1-7)$, however, has been shown to produce both antinociception (Hall and Stewart, 1986), which appears to be mediated by activation of $\mu$-type opioid receptors (Goettl and Larson, 1991), as well as hyperalgesic activity (Cridland and Henry, 1988). While the variability of effects produccd by SP(1-7) may reflect differences in species, doses, routes of administration, or time of testing, our dose-response data suggest the possibility of a dual action of SP(1-7) on KA-induced behavioral responses. A similar impact on the ability of $\mathrm{SP}(1-7)$ to alter nociceptive activity may result in the enhancement of nociceptive activity at lower doses of SP(1-7) and the inhibition of nociception by higher doses.

SP $\mathrm{NH}_{2}$-terminal activity has been previously reported to consist primarily of inhibitory effects. Behavioral desensitization to repeated injections of SP, for example, may be brought about by an accumulation of $\mathrm{NH}_{2}$-terminal metabolites of SP (Larson, 1988) based on (1) the inability of repeated injections of SP(3-11) to exhibit behavioral desensitization (Igwe et al., 1990a), (2) the ability of SP(1-7) to inhibit the excitatory effects of SP (Igwe et al., 1990a), and (3) the strong correlation between the accumulation of $\mathrm{NH}_{2}$-terminal fragments of SP and development of descnsitization (Igwe ct al., 1990b). The major metabolite of SP appears to be the $\mathrm{NH}_{2}$-terminal heptapeptide SP(1-7) (Nyberg et al., 1984; Sakurada et al., 1985). High-affinity binding of ${ }^{3} \mathrm{H}-\mathrm{SP}(1-7)$ in mouse brain and spinal cord tissue preparations suggests the existence of a non-neurokinin SP receptor that may account for the activity of $\mathrm{SP} \mathrm{NH}_{2}$-terminal fragments (Igwe et al., 1990c).

Together, our data support the hypothesis that SP is released in response to KA activity in the mouse spinal cord, and that endogenously released SP then facilitates subsequent KA-induced behavior by an action mediated primarily by the $\mathrm{SP} \mathrm{NH} \mathrm{N}_{2}$ terminal receptor and only to a minor extent by the neurokinin receptor. While the $\mathrm{NH}_{2}$-terminal fragment of $\mathrm{SP}$ is sufficient to elicit the potentiative effect, metabolism of SP to $\mathrm{NH}_{2}-$ terminal peptide fragments does not appear to be necessary for the production of $\mathrm{NH}_{2}$-terminus-mediated effects. We propose that this modulation of EAA-induced activity and the $\mathrm{NH}_{2}$ terminus of SP may prove to be important in a variety of systems including pain transmission.

\section{References}

Aanonsen LM, Wilcox GL (1987) Nociceptive action of excitatory amino acids in the mouse: effects of spinally administered opioids, phencyclidine and sigma agonists. J Pharmacol Exp Ther 243:9-19.

Besson J, Chaouch A (1987) Peripheral and spinal mechanism of nociception. Physiol Rev 67:67-185.

Cridland RA, Henry JL (1988) N-and C-terminal fragments of substance P: spinal effects in the rat tail flick test. Brain Res Bull 20:429432.

De Biasi S, Rustioni A (1988) Glutamate and substance P coexist in primary afferent terminals in superficial laminae of spinal cord Proc. Natl Acad Sci USA 85:7820-7824.

Dougherty PM, Willis WD (1991) Enhancement of spinothalamic neuron responses to chemical and mechanical stimuli following combined micro-iontophoretic application of $N$-methyl-D-aspartate and substance $P$. Pain 47:85-93.

Engberg G, Svensson TH, Rosell S, Folkers K (1981) A synthetic peptide as an antagonist of substance $P$. Nature 293:222-223.

Goettl VM, Larson AA (1991) Substance P(1-7) spinal antinociception 
and interaction with the $\beta$-FNA-sensitive opiate receptor subtype. Soc Neurosci Abstr 17:1373.

Hall ME, Stewart JM (1983) Substance P and behavior: opposing effects of N-terminal and C-terminal fragments. Peptides 4:763-768.

Hall ME, Stewart JM (1984) Modulation of isolation-induced fighting by $\mathrm{N}$ - and $\mathrm{C}$-terminal analogs of substance $\mathrm{P}$ : evidence for multiple recognition sites. Peptides 5:85-89.

Hall ME, Stewart JM (1986) Substance P and antinociception. Peptides 4:31-35.

Hylden JLK, Wilcox GL (1980) Intrathecal morphine in mice: a new technique. Eur J Pharmacol 67:313-316.

Hylden JLK, Wilcox GL (1981) Intrathecal substance P elicits a caudally-directed biting and scratching behavior in mice. Brain Res 217 : 212-215.

Igwe OJ, Sun X, Larson AA (1990a) Role of substance $P$ amino terminal metabolites in substance P-induced desensitization in mice. Neuroscience 36:535-542.

Igwe OJ, Sun X, Larson AA (1990b) Correlation of substance Pinduced desensitization with substance $P$ amino terminal metabolites in the mouse spinal cord. Peptides 11:817-825.

Igwe OJ, Sun X, Larson AA (1990c) Specific binding of substance $P$ aminoterminal heptapeptide [SP(1-7)] to mouse brain and spinal cord membranes. J Neurosci 10:3653-3663.

Larson AA (1988) Desensitization to intrathecal substance $P$ in mice: possible involvement of opioids. Pain 32:367-374.

Larson AA, Beitz AJ (1988) Glycine potentiates strychnine-induced convulsions: role of NMDA receptors. J Neurosci 8:3822-3826.

Lembeck $F$ (1953) Zur frage der zentralen ubcrtragung afferenter impulse. III. Mitteilung. Das vorkommen und die bedentung der substanz $\mathbf{P}$ in den dorsalen wurzeln des ruckenmarks. Naunyn Schmiedebergs Arch Exp Pathol Pharmacol 219:197-213.

Matsumura H, Sakurada T, Hara A, Sakurada S, Kisara K (1985)
Characterization of the hyperalgesic effect induced by intrathecal iniection of substance $P$. Neuropharmacology 24:421-426.

Murray CW, Skilling SR, Smullin DH, Larson AA (1990) Release of substance $\mathbf{P}$ in the dorsal lumbar spinal cord of the conscious, freely moving rat: stimulation by kainic acid, capsaicin and substance $\mathbf{P}(1$ 7). Soc Neurosci Abstr 16:161.

Nyberg F, Le Greves P, Sundqvist S, Terenius L (1984) Characterization of substance $P(1-7)$ and (1-8) generating enzyme in human cerebralspinal fluid. Biochem Biophys Res Commun 125:244-250.

Piercy MF, Dobry PJK, Schoeder LA, Einspahr RJ (1981) Behavioral evidence that substance $P$ may be a spinal cord sensory neurotransmitter. Brain Res 210:407-412.

Sakurada T, Le Greves P, Stewart J, Terenius L (1985) Measurement of substance $P$ metabolites in rat CNS. J Neurochem 44:718-722.

Sakurada T, Yamada T, Sakurada S, Kisara K, Ohba M (1989) Substance $\mathrm{P}$ analogues containing $\mathrm{D}$-histidine antagonize the behavioral effects of intrathecally co-administered substance $P$ in mice. Eur $J$ Pharmacol 174:153-160.

Salt TE, Hill RG (1983) Transmitter candidates of somatosensory primary afferent fibers. Neuroscience 10:1083-1 103.

Skilling SR, Smullin DH, Beitz AJ, Larson AA (1988) Extracellular amino acid concentrations in the dorsal spinal cord of freely moving rats following veratridine and nociceptive stimulation. J Neurochem 51:127-132.

Stewart JM, Hall ME, Harkins J, Frederickson RCA, Terenius L, Hokfelt T, Krivoy WA (1982) A fragment of substance $P$ with specific central activity: SP(1-7). Peptides 3:851-857.

Sun X, Larson AA (1991) Behavioral sensitization to kainic acid and quisqualic acid in mice: comparison to NMDA and substance $P$ responses. J Neurosci 11:3111-3123.

Takagi H (1984) Experimental pain and neuropeptides. Clin Ther 7: $35-47$. 